\title{
The Importance of Physical Activity Exercise among Older People
}

\author{
Birgitta Langhammer $\mathbb{D}^{1},{ }^{1}$ Astrid Bergland, ${ }^{2}$ and Elisabeth Rydwik ${ }^{3,4}$ \\ ${ }^{1}$ Oslo Metropolitan University, Faculty of Health Sciences and Sunnaas Rehabilitation Hospital, Oslo, Norway \\ ${ }^{2}$ Oslo Metropolitan University, Faculty of Health Sciences, Oslo, Norway \\ ${ }^{3}$ Karolinska Institutet, Department of Neurobiology, Care Sciences and Society, Division of Physiotherapy, Huddinge, Sweden \\ ${ }^{4}$ Stockholm County Council, Research and Development Unit for the Elderly, Järfälla, Sweden
}

Correspondence should be addressed to Birgitta Langhammer; birgitta.langhammer@oslomet.no

Received 22 November 2018; Accepted 22 November 2018; Published 5 December 2018

Copyright (c) 2018 Birgitta Langhammer et al. This is an open access article distributed under the Creative Commons Attribution License, which permits unrestricted use, distribution, and reproduction in any medium, provided the original work is properly cited.

In this special issue of BioMed Research International, the focus is on lifestyle and in particular physical activity (PA) as a driver for a healthy and long life for older people.

As populations continue to extend life expectancy, a central concern is whether the added time comprises years of healthy life and promotes a high health-related quality of life into old age. $P A$ is defined as any bodily movement produced by skeletal muscles that result in energy expenditure. PA encompasses exercise, sports, and physical activities performed as part of daily living, occupation, leisure, or active transportation. Exercise is a subcategory of PA that is planned, structured, and repetitive and that has as a final or intermediate objective for improvement or maintenance of physical fitness. Physical function is the capacity of an individual to perform the physical activities of daily living. Physical function reflects motor function and control, physical fitness, and habitual PA [1].

$\mathrm{PA}$ is a protective factor for noncommunicable diseases such as cardiovascular disease, stroke, diabetes, and some types of cancer [2] and PA is associated with improved mental health [3], delay in the onset of dementia [4], and improved quality of life and wellbeing $[5,6]$. The health benefits of PA are well documented with higher levels and greater frequency of PA being associated with reduced risk and improved health in a number of key areas [7].

The dose of PA or exercise is described by the duration, frequency, intensity, and mode [8]. For optimal effects, the older person must adhere to the prescribed exercise program and follow the overload principle of training, i.e., to exercise near the limit of the maximum capacity to challenge the body systems sufficiently, to induce improvements in physiological parameters such as VO2max and muscular strength [1].

Improvements in mental health, emotional, psychological, and social well-being and cognitive function are also associated with regular PA. Despite these health benefits, PA levels amongst older adults remain below the recommended $150 \mathrm{~min} /$ week [9]. The crude global prevalence of physical inactivity is $21.4 \%$ [10]. This translates to one in every four to five adults being physically inactive, or with activity levels lower than the current recommendations from WHO [11]. Inactivity and aging increase the risk of chronic disease, and older people often have multiple chronic conditions (NFH, 2010). The exercise recommendations from WHO include both aerobic exercise and strength exercise as well as balance exercises to reduce the risk of falls. If older adults cannot follow the guidelines because of chronic conditions, they should be as active as their ability and conditions allow [12]. It is important to note that the recommended amount of PA is in addition to routine activities of daily living like self-care, cooking, and shopping, to mention a few.

Inactivity is associated with alterations in body composition resulting in an increase in percentage of body fat and a concomitant decline in lean body mass. Thus, significant loss in maximal force production takes place with inactivity. Skeletal muscle atrophy is often considered a hallmark of aging and physical inactivity. Sarcopenia is defined as low muscle mass in combination with low muscle strength and/or low physical performance [13]. Consequently, low physical 
performance and dependence in activities of daily living is more common among older people [14, 15]. However, strength training has been shown to increase lean body mass [16], improve physical performance $[17,18]$, and to a lesser extent have a positive effect on self-reported activities of daily living [18]. These aspects are at focus in the papers of K. Kropielnicka et al. "Influence of the Physical Training on Muscle Function and Walking Distance in Symptomatic Peripheral Arterial Disease in Elderly" as well as G. Piastra et al. "Effects of Two Types of 9-Month Adapted Physical Activity Program on Muscle Mass, Muscle Strength, and Balance in Moderate Sarcopenic Older Women.”

Participation in PA and exercise can contribute to maintaining quality of life, health, and physical function and reducing falls [19-21] among older people in general and older people with morbidities in particular. The increased attention to the relationship between exercise and HRQOL in older adults over the last decade is reflected in a recent review, which showed that a moderate PA level combining multitasking exercise components had a positive effect on activities in daily living, highlighting the importance of physical, mental, and social demands [22]. To reduce falls, balance training is also recommended to be included in physical exercise programs for older adults [12]. Exercise has also been shown to reduce falls with $21 \%$, with a greater effect of exercise programs including challenging balance activities for more than 3 hours/week [23].

The gender perspective and motivators for fall prevention are at focus in M. Sandlund et al. qualitative study "Gender Perspective on Older People's Exercise Preferences and Motivators in the Context of Falls Prevention: A Qualitative Study," in this special issue.

Exercise training in older people has been associated with health benefits such as decreased cardiovascular mortality [24]. Explanatory mechanism likely to be involved following exercise was a change in the cardiac autonomic balance producing an increase, or a relative dominance, of the vagal component [25]. Furthermore, endurance exercise training in older people decreases resting and submaximal exercise heart rate and systolic and diastolic blood pressure and increases stroke volume [26]. This is especially notable during peak effort in which stroke volume, cardiac output, contractility, and oxygen uptake are increased, while total peripheral resistance and systolic and diastolic blood pressure decreased. Thus lowering after-load in the heart muscle, which in turn facilitates left ventricular systolic and diastolic function, emphasizes the importance of high intensity training also for the elderly. E. Tamuleviciute-Prasciene et al. focus on the frail elderly individuals and exercise in their contribution "Frailty and Exercise Training: How to Provide Best Care after Cardiac Surgery or Intervention for Elder Patients with Valvular Heart Disease."

Exercise may also have benefits for the brain centers that support executive control. It may be that strong executive functioning in itself may facilitate consistency for this challenging activity. Poor executive control has been associated with lower self-reported PA rates over a 2-year period [27, 28]. The executive control's contribution to PA has been found to be $50 \%$ greater in magnitude than the contribution of PA to subsequent changes in executive control [29]. In the paper of M. A. McCaskey et al. "Making More of IT: Enabling Intensive MOtor Cognitive Rehabilitation Exercises in Geriatrics Using INFORMATION Technology Solutions," the authors also include new technology to enhance and maintain exercise in cognitive rehabilitation.

In order to attain a high level of cardiorespiratory fitness, it is recommended to be physically active for 6 months or longer. These recommendations may also be applied to balance exercises in order to reduce falls [23]. Many elderly individuals are incapable of sustaining activities for this long on their own. Successful maintenance of PA typically requires substantial support and supervision. Even then, a high percentage of people drop out due to difficulties negotiating everyday costs of activity participation like scheduling conflicts and competing sedentary activities or health issues. This issue is highlighted in the study of T. Adachi et al. "Predicting the Future Need of Walking Device or Assistance by Moderate to Vigorous Physical Activity: A 2-Year Prospective Study of Women Aged 75 Years and Above."

In addition, reduced bodily functions can make it difficult for elderly persons to maintain exercise under different environmental circumstances, which is demonstrated in the contribution of B. N. Balmain et al. "Aging and Thermoregulatory Control: The Clinical Implications of Exercising under Heat Stress in Older Individuals."

In this special issue, we have included papers that focus on the aging process and PA in a broad perspective, focusing on different aspects on PA, exercise, and older people. PA and exercise play an important role in the primary, secondary, and tertiary prevention, in the management of diseases, to counteract sarcopenia and falls as well as improving physical performance and activities of daily living, as these papers illustrate.

Promoting exercise among the older population is an important public health and clinical issue. A core issue is how to get older people with comorbidities to exercise.

\section{Conflicts of Interest}

The authors declare that they have no conflicts of interest.

Birgitta Langhammer
Astrid Bergland
Elisabeth Rydwik

\section{References}

[1] C. E. Garber, B. Blissmer, M. R. Deschenes et al., "Quantity and quality of exercise for developing and maintaining cardiorespiratory, musculoskeletal, and neuromotor fitness in apparently healthy adults: guidance for prescribing exercise," Medicine \& Science in Sports \& Exercise, vol. 43, no. 7, pp. 1334-1359, 2011.

[2] World Health Organization, "PA for health. More active people for a healthier world: draft global action plan on PA 2018- 2030," Vaccine, 2018.

[3] F. B. Schuch, D. Vancampfort, J. Richards, S. Rosenbaum, P. B. Ward, and B. Stubbs, "Exercise as a treatment for depression: A meta-analysis adjusting for publication bias," Journal of Psychiatric Research, vol. 77, pp. 42-51, 2016. 
[4] G. Livingston, A. Sommerlad, V. Orgeta et al., "Dementia prevention, intervention, and care," The Lancet, vol. 390, no. 10113, pp. 2673-2734, 2017.

[5] P. Das and R. Horton, "Rethinking our approach to physical activity," The Lancet, vol. 380, no. 9838, pp. 189-190, 2012.

[6] F. E. F. Camboim, M. O. Nóbrega, R. M. B. Davim et al., "et alenefits of PA in the third age for the quality of life," $J$ Nurs Recife, vol. 11, no. 6, pp. 2415-22, 2017.

[7] S. Musich, S. S. Wang, K. Hawkins, and C. Greame, "The Frequency and Health Benefits of Physical Activity for Older Adults," Population Health Management, vol. 20, no. 3, pp. 199207, 2017.

[8] D. W. Brown, D. R. Brown, G. W. Heath et al., "Associations between Physical Activity Dose and Health-Related Quality of Life," Medicine \& Science in Sports \& Exercise, vol. 36, no. 5, pp. 890-896, 2004.

[9] E. R. Boulton, M. Horne, and C. Todd, "Multiple influences on participating in physical activity in older age: Developing a social ecological approach," Health Expectations, vol. 21, no. 1, pp. 239-248, 2018.

[10] S. C. Dumith, P. C. Hallal, R. S. Reis, and H. W. Kohl, "Worldwide prevalence of physical inactivity and its association with human development index in 76 countries," Preventive Medicine, vol. 53, no. 1-2, pp. 24-28, 2011.

[11] WHO, Global Recommendations on PA for Health, 2010b, http:// www.who.int/dietphysicalactivity/factsheet_olderadults/en/index.htmlPA for Health from.

[12] W. J. Chodzko-Zajko, D. N. Proctor, M. A. Fiatarone Singh et al., "Exercise and physical activity for older adults," Medicine \& Science in Sports \& Exercise, vol. 41, no. 7, pp. 1510-1530, 2009.

[13] A. J. Cruz-Jentoft, J. P. Baeyens, J. M. Bauer et al., "Sarcopenia: European consensus on definition and diagnosis," Age and Ageing, vol. 39, no. 4, Article ID afq034, pp. 412-423, 2010.

[14] G. Idland, E. Rydwik, M. C. Småstuen, and A. Bergland, "Predictors of mobility in community-dwelling women aged 85 and older," Disability and Rehabilitation, vol. 35, no. 11, pp. 881887, 2013.

[15] B.-M. Sjölund, A. Wimo, M. Engström, and E. Von Strauss, "Incidence of ADL disability in older persons, physical activities as a protective factor and the need for informal and formal care -results from the snac-n project," PLoS ONE, vol. 10, no. 9, 2015.

[16] M. D. Peterson, A. Sen, and P. M. Gordon, "Influence of resistance exercise on lean body mass in aging adults: a metaanalysis," Medicine \& Science in Sports \& Exercise, vol. 43, no. 2, pp. 249-258, 2011.

[17] P. Lopez, R. S. Pinto, R. Radaelli et al., "Benefits of resistance training in physically frail elderly: a systematic review," Aging Clinical and Experimental Research, vol. 30, no. 8, pp. 889-899, 2018.

[18] M. Giné-Garriga, M. Roqué-Fíguls, L. Coll-Planas, M. SitjàRabert, and A. Salvà, "Physical exercise interventions for improving performance-based measures of physical function in community-dwelling, frail older adults: a systematic review and meta-analysis," Archives of Physical Medicine and Rehabilitation, vol. 95, no. 4, pp. 753-769, 2014.

[19] L. D. Gillespie, M. C. Robertson, W. J. Gillespie et al., "Interventions for preventing falls in older people living in the community," Cochrane Database of Systematic Reviews, no. 9, 2012.

[20] F. El-Khoury, B. Cassou, M.-A. Charles, and P. DargentMolina, "The effect of fall prevention exercise programmes on fall induced injuries in community dwelling older adults: Systematic review and meta-analysis of randomised controlled trials," BMJ, vol. 347, Article ID f6234, 2013.

[21] A. C. Tricco, S. M. Thomas, A. A. Veroniki et al., "Comparisons of interventions for preventing falls in older adults: A systematic review and meta-analysis," Journal of the American Medical Association, vol. 318, no. 17, pp. 1687-1699, 2017.

[22] C. E. Roberts, L. H. Phillips, C. L. Cooper, S. Gray, and J. L. Allan, "Effect of Different Types of Physical Activity on Activities of Daily Living in Older Adults: Systematic Review and Meta-Analysis," Journal of Aging and Physical Activity, vol. 25, no. 4, pp. 653-670, 2017.

[23] C. Sherrington, Z. A. Michaleff, N. Fairhall et al., "Exercise to prevent falls in older adults: An updated systematic review and meta-analysis," British Journal of Sports Medicine, vol. 51, no. 24, pp. 1749-1757, 2017.

[24] J. A. Laukkanen, S. Kurl, R. Salonen, R. Rauramaa, and J. T. Salonen, "The predictive value of cardiorespiratory fitness for cardiovascular events in men with various risk profiles: A prospective population-based cohort study," European Heart Journal, vol. 25, no. 16, pp. 1428-1437, 2004.

[25] N. Eynon, M. Sagiv, O. Amir, D. Ben-Sira, E. Goldhammer, and R. Amir, "The effect of long-term $\beta$-adrenergic receptor blockade on the oxygen delivery and extraction relationship in patients with coronary artery disease," Journal of Cardiopulmonary Rehabilitation and Prevention, vol. 28, no. 3, pp. 189194, 2008.

[26] D. K. McGuire, B. D. Levine, J. W. Williamson et al., "A 30-year follow-up of the Dallas bed rest and training study: I. Effect of age on the cardiovascular response to exercise," Circulation, vol. 104, no. 12, pp. 1350-1357, 2001.

[27] M. Daly, D. McMinn, and J. L. Allan, "A bidirectional relationship between physical activity and executive function in older adults," Frontiers in Human Neuroscience, vol. 8, 2015.

[28] O. Dupuy, C. J. Gauthier, S. A. Fraser et al., "Higher levels of cardiovascular fitness are associated with better executive function and prefrontal oxygenation in younger and older women," Frontiers in Human Neuroscience, vol. 9, 2015.

[29] J. R. Best, L. S. Nagamatsu, and T. Liu-Ambrose, "Improvements to executive function during exercise training predict maintenance of physical activity over the following year," Frontiers in Human Neuroscience, vol. 8, article no. 353, 2014. 


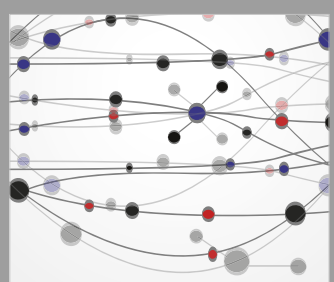

The Scientific World Journal
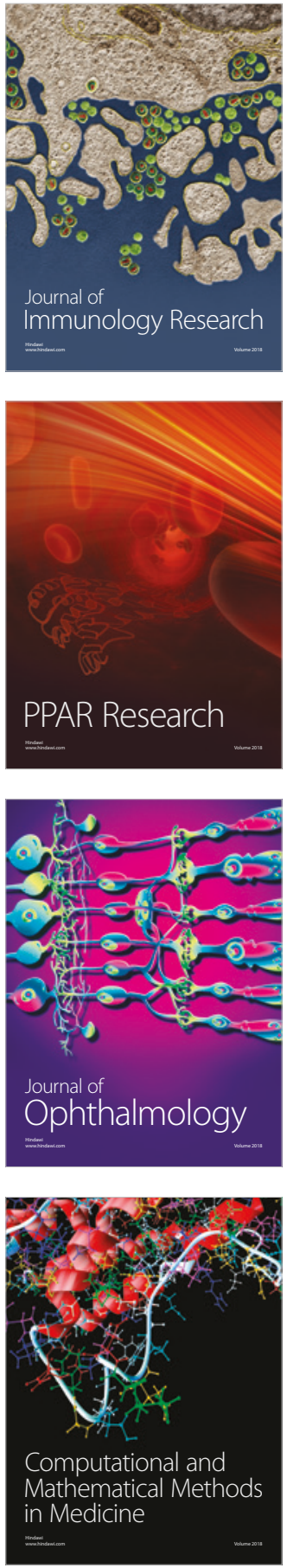

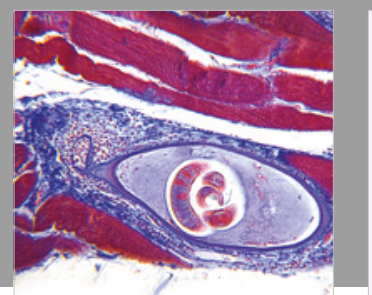

Gastroenterology Research and Practice

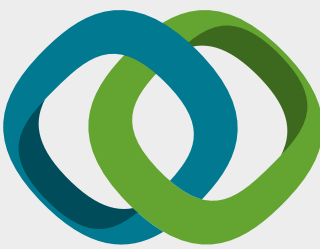

\section{Hindawi}

Submit your manuscripts at

www.hindawi.com
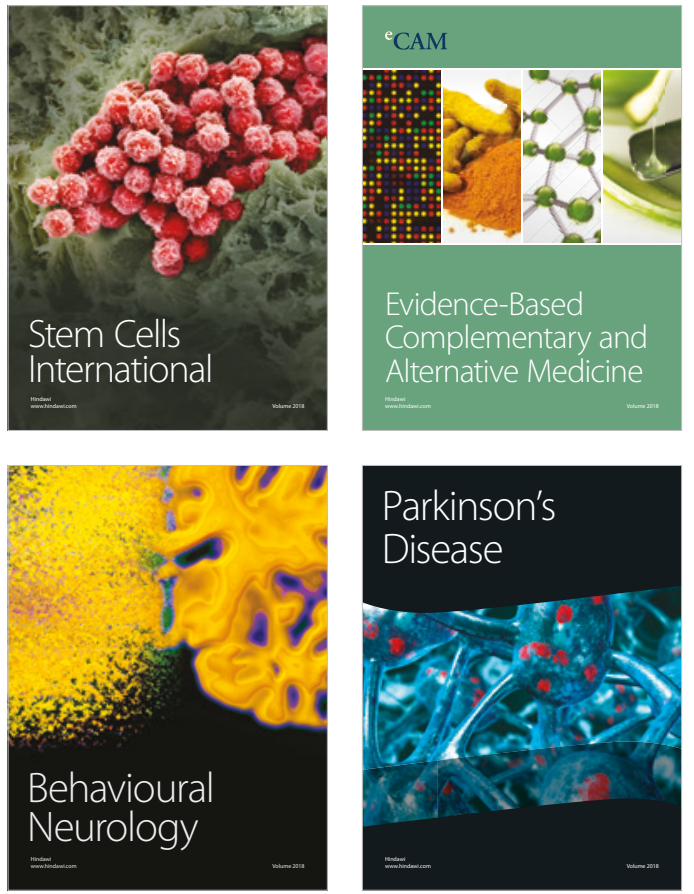

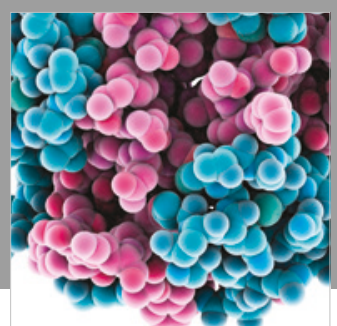

ournal of

Diabetes Research

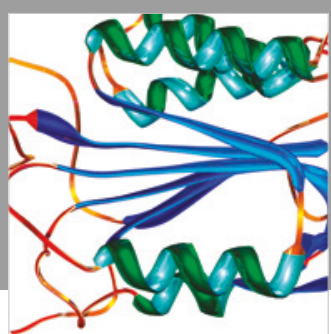

Disease Markers
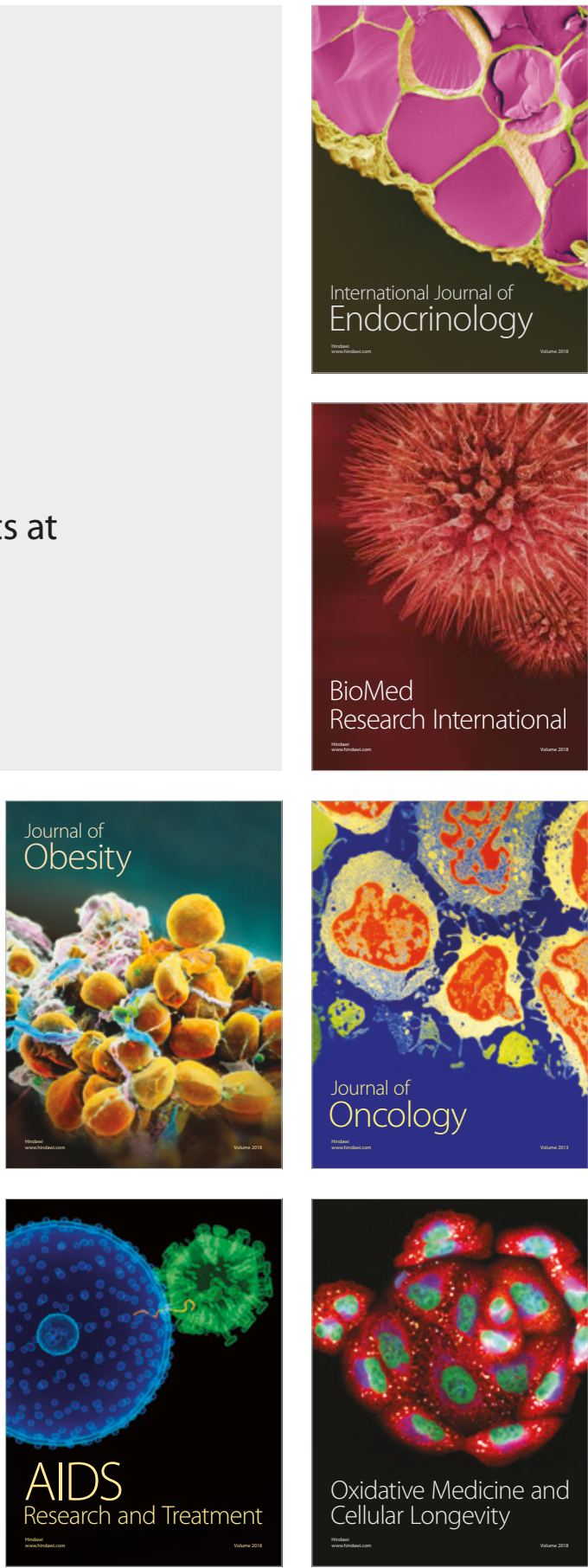